\title{
Supplementary Material to “A Bayesian Approach to Modeling Multivariate Multilevel Insurance Claims in the Presence of Unsettled Claims"
}

\author{
Marie-Pier Côté* ${ }^{\text {, Christian Genest }}{ }^{\dagger, \ddagger}$ and David A. Stephens ${ }^{\dagger}$
}

\begin{abstract}
This Online Supplement contains the expression of the moment generating function of the Fernández-Steel skewed normal, the derivation of the informative prior distribution on the correlation matrix and the prior specification, the transition probabilities between types varying with development periods, the regression models for the missing covariates, the MCMC algorithms, and the summary of the posterior distributions of the model parameters.
\end{abstract}

MSC 2010 subject classifications: Primary 62F15, 62P05; secondary 62H05.

Keywords: Bayesian model, censored data, copula, correlation, Fernández-Steel skewed normal, imputation, insurance claim, multinomial and logistic regression.

\section{Introduction}

This document complements the paper titled "A Bayesian Approach to Modeling Multivariate Multilevel Insurance Claims in the Presence of Unsettled Claims," by M.-P. Côté, C. Genest, and D.A. Stephens.

Section 2 concerns the Fernández-Steel skewed normal model. A formula for its cumulative distribution function is given, and an apparently new expression for the corresponding moment generating function is stated and proved in Proposition 1.

Section 3 details the development of the informative prior for the correlation matrix, documents the choice of prior parameters, and compares the prior and posterior joint distributions of the dependence parameters.

Section 4 complements Section 4.1 in the main paper. It describes the approach used for estimating the transition probabilities for the imputation model of the censored types and presence of external Expenses, which can be found in Tables 1 and 2.

Section 5 provides details on the proportional odds regression model used to impute the missing pain levels in the type model. The priors for the missing pain levels, income indemnity level, and claimant age used in the amounts model are also reported.

*École d'actuariat, Université Laval, 2425, rue de l'Agriculture, Québec (Québec) Canada G1V 0A6 marie-pier.cote@act.ulaval.ca

${ }^{\dagger}$ Department of Mathematics and Statistics, McGill University, 805, rue Sherbrooke ouest, Montréal (Québec) Canada H3A 0B9 christian.genest@mcgill.ca, david.stephens@mcgill.ca

‡Corresponding author 
Section 6 outlines the MCMC algorithm for the parameters of the type and presence of external Expenses parameters. The posterior mean and standard deviations of the parameters are shown in Tables 7 and 8 .

Finally, Section 7 outlines the MCMC algorithm for the parameters of the amounts distribution, including the marginal log-skewed normal regressions and the copula parameters. The posterior mean and standard deviation of the marginal parameters are in Tables 9-10, while those of the dependence structure are in the main paper.

\section{Fernández-Steel skewed normal model}

The Fernández-Steel skewed normal density and parameters are specified in Section 3.3 of the main paper. The density is shown in Figure 1 below for various values of $\xi$. The cumulative distribution function, obtained by a change of variable, is given by

$$
F(y \mid \mu, \sigma, \xi)=\frac{2 \xi^{\operatorname{sign}\left(y \sigma^{*}+\mu^{*}\right)}}{\xi+1 / \xi} \Phi\left\{\frac{y \sigma^{*}+\mu^{*}}{\xi^{\operatorname{sign}\left(y \sigma^{*}+\mu^{*}\right)}}\right\}+\frac{\xi^{-1}-\xi^{\operatorname{sign}\left(y \sigma^{*}+\mu^{*}\right)}}{\xi+1 / \xi},
$$

where $\Phi$ refers to the $\mathcal{N}(0,1)$ cumulative distribution function.

The following result, which is apparently new, guarantees the existence of the moments of the paid amounts on the original scale.

Proposition 1. The moment generating function of the Fernández-Steel skewed normal distribution is given, for all $t \in \mathbb{R}$, by

$$
M_{Y}(t ; \mu, \sigma, \xi)=\frac{2}{\xi+1 / \xi} \exp \left(-\frac{\mu^{*} t}{\sigma^{*}}\right) \sum_{\ell \in\{-1,1\}} \xi^{\ell} \exp \left\{\frac{t^{2} \xi^{2 \ell}}{2\left(\sigma^{*}\right)^{2}}\right\} \Phi\left(-\frac{t \xi^{\ell}}{\sigma^{*}}\right) .
$$

Proof. The moment generating function can be obtained by computing

$$
\mathrm{E}\left(e^{t Y}\right)=\int_{-\infty}^{\infty} e^{t y} \frac{2 \sigma^{*}}{\xi+1 / \xi} \phi\left\{\frac{y \sigma^{*}+\mu^{*}}{\xi^{\operatorname{sign}\left(y \sigma^{*}+\mu^{*}\right)}}\right\} \mathrm{d} y
$$

First, observe that it can be split into two parts as follows:

$$
\begin{aligned}
\mathrm{E}\left(e^{t Y}\right)=\frac{2 \sigma^{*}}{\xi+1 / \xi}\left[\int_{-\infty}^{-\mu^{*} / \sigma^{*}} \frac{1}{\sqrt{2 \pi}}\right. & \exp \left\{t y-\frac{\xi^{2}}{2}\left(y \sigma^{*}+\mu^{*}\right)^{2}\right\} \mathrm{d} y \\
& \left.+\int_{-\mu^{*} / \sigma^{*}}^{\infty} \frac{1}{\sqrt{2 \pi}} \exp \left\{t y-\frac{1}{2 \xi^{2}}\left(y \sigma^{*}+\mu^{*}\right)^{2}\right\} \mathrm{d} y\right] .
\end{aligned}
$$

By completing the square, the first integral can be rewritten as

$$
\exp \left\{\frac{t^{2}}{2\left(\sigma^{*}\right)^{2} \xi^{2}}-\frac{\mu^{*} t}{\sigma^{*}}\right\} \frac{1}{\xi \sigma^{*}}
$$




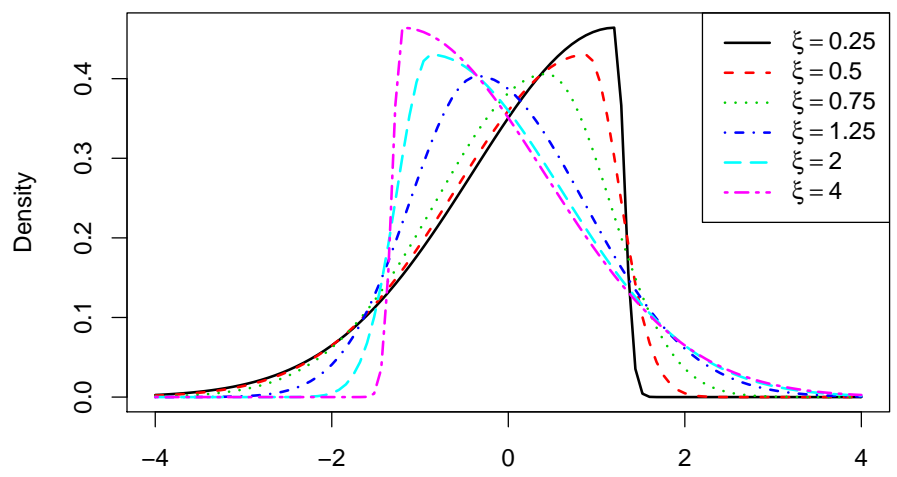

Figure 1: Density of the $\mathcal{S N}(0,1, \xi)$ distribution for various values of $\xi$.

$$
\times \int_{-\infty}^{-\mu^{*} / \sigma^{*}} \frac{1}{\sqrt{2 \pi} /\left(\xi \sigma^{*}\right)} \exp \left[-\frac{\left(\sigma^{*}\right)^{2} \xi^{2}}{2}\left[y-\left\{\frac{t}{\xi^{2}\left(\sigma^{*}\right)^{2}}-\frac{\mu^{*}}{\sigma^{*}}\right\}\right]^{2}\right] \mathrm{d} y
$$

which can be expressed in terms of the $\mathcal{N}(0,1)$ distribution function as

$$
\exp \left\{\frac{t^{2}}{2\left(\sigma^{*}\right)^{2} \xi^{2}}-\frac{\mu^{*} t}{\sigma^{*}}\right\} \frac{1}{\xi \sigma^{*}} \Phi\left(-\frac{t}{\xi \sigma^{*}}\right) .
$$

Similarly, completing the square in the second integral and using the symmetry of $\Phi$, one finds

$$
\exp \left\{\frac{\xi^{2} t^{2}}{2\left(\sigma^{*}\right)^{2}}-\frac{\mu^{*} t}{\sigma^{*}}\right\} \frac{\xi}{\sigma^{*}} \Phi\left(\frac{\xi t}{\sigma^{*}}\right) .
$$

The desired expression is obtained upon bringing the two parts together. If $\xi=1$, then $\mu^{*}=-\mu / \sigma$ and $\sigma^{*}=1 / \sigma$ and the expression reduces to the well-known moment generating function of the symmetric Gaussian distribution.

\section{Prior on the correlation matrix}

As noted by Barnard et al. (2000), the Jacobian of the transformation $W \mapsto(R, A)$ is $2^{k}\left(a_{1}^{k} \times \cdots \times a_{k}^{k}\right)$. If $k=1$, this is trivial as $W=a_{1}^{2}$, so the Jacobian is $\left|2 s_{1}\right|$. To derive the Jacobian for general $k$, note that the matrix of partial derivatives can be arranged as a lower triangular matrix, so that the determinant is simply the product of the diagonal elements. Also, if $W$ has an inverse Wishart distribution with positive definite scale matrix $\Psi$, then

$$
f(W) \propto|W|^{-(\nu+k+1) / 2} \exp \left\{-\frac{1}{2} \operatorname{tr}\left(\Psi W^{-1}\right)\right\} .
$$


As $W=A R A$ and the matrix $A$ is diagonal, $|W|=\left(a_{1} \times \cdots \times a_{k}\right)^{2}|R|$ and

$$
f(S, R) \propto|R|^{-(\nu+k+1) / 2} \exp \left[-\frac{1}{2} \operatorname{tr}\left\{\Psi \operatorname{diag}\left(1 / a_{i}\right) R^{-1} \operatorname{diag}\left(1 / a_{i}\right)\right\}\right] \prod_{i=1}^{k} a_{i}^{-(\nu+1)} .
$$

Consider the case where $\Psi$ is a symmetric matrix with diagonal elements equal to 1 and off-diagonal elements equal to $\rho$. Then $\operatorname{diag}\left(1 / a_{i}\right) \Psi \operatorname{diag}\left(1 / a_{i}\right)$ has $i$ th diagonal element $1 / a_{i}^{2}$ and element $(i, j)$ equal to $\rho /\left(a_{i} a_{j}\right)$. So

$$
\operatorname{tr}\left\{\Psi \operatorname{diag}\left(1 / a_{i}\right) R^{-1} \operatorname{diag}\left(1 / a_{i}\right)\right\}=\sum_{i=1}^{k} \frac{r^{i i}}{a_{i}^{2}}+2 \rho \sum_{i=1}^{k} \sum_{j=i+1}^{k} \frac{r^{i j}}{a_{i} a_{j}},
$$

where $r^{i j}$ is the $(i, j)$ th element of $R^{-1}$. The distribution of $R$ simplifies nicely when $\rho=0$, using the transformation $u_{i}=r^{i i} /\left(2 a_{i}^{2}\right)$ to integrate out the positive nuisance parameters $a_{1}, \ldots, a_{k}$. One finds

$$
f(R) \propto|R|^{-(\nu+k+1) / 2} \prod_{i=1}^{k}\left(r^{i i}\right)^{-\nu / 2},
$$

which is the case of the standard inverse Wishart $\Psi=I$ considered by Barnard et al. (2000). When $\nu=k+1$, each correlation is uniform on the interval $(-1,1)$, making the prior non-informative on the marginals. When $\nu>k+1$, the margin is centered at 0 and thus appropriate only when shrinking the correlations towards 0 is desirable, which is not the case in the application considered in the main paper.

For insurance claims data, it is natural to expect positive association between the amounts paid in relation to a given claim. Based on previous experience from this insurance company, Kendall's tau should be close to $1 / 3$, which corresponds to a correlation of $1 / 2$ for the Student $t$ copula. The use of an informative prior on the correlation matrix thus seems appropriate. As $a_{1}, \ldots, a_{k}$ cannot be integrated out easily, they are introduced as latent variables on which the algorithm does not learn. The joint prior is

$$
\pi_{0}(A, R) \propto|R|^{-(\nu+k+1) / 2} \exp \left(-\frac{1}{2} \sum_{i=1}^{k} \frac{r^{i i}}{a_{i}^{2}}+\sum_{i=1}^{k} \sum_{j=i+1}^{k} \frac{\rho r^{i j}}{a_{i} a_{j}}\right) \prod_{i=1}^{k} a_{i}^{-(\nu+1)} .
$$

Figure 2 below shows the estimated marginal densities for different choices of degrees of freedom $\nu$ and prior correlation $\rho$, based on realizations from the distribution of the random correlation matrix $R$ induced by $W$.

We use $\rho=1 / 2$ and $\nu=15$, putting most of the weight (around 97\%) on positive correlations that are plausible. With 30,000 realizations, the moments may be estimated with high precision. The expectation is 0.48 , the standard deviation is 0.22 , and the median is 0.51 .

The dependence between the correlations generated by this model seems to be partly induced by the positive definiteness constraint: association between correlations with 

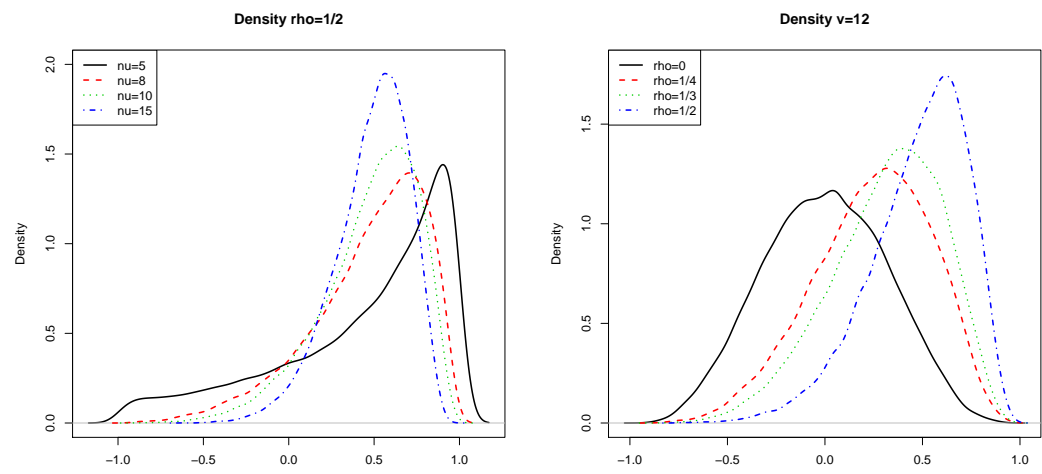

Figure 2: Approximate marginal densities of the correlation induced by a $4 \times 4$ Inverse Wishart $(\Psi, \nu)$ random matrix, for different values of $\nu$ (left) and $\rho$ (right).

common indices is larger, as illustrated in the left panel of Figure 3 herein. Figure 3 compares Kendall's tau matrices induced by the prior and posterior for $\Lambda$, showing that prior dependence between $\rho_{1,2}, \ldots, \rho_{3,4}$ is stronger than the posterior association.

\section{Transition probabilities for type and presence of expenses for open files}

As explained in Section 4.1 of the main paper, the types for open claims may change before settlement. The probability of staying in the current type varies with the development period and differs for minor and major or catastrophic claims.

The estimated probabilities are shown in Table 1 below. They were obtained using the development data for claims that were closed before evaluation date. To reduce possible bias, claims incurred in years 2004 to 2006 that closed before year 2007 were dropped to offset the effect of including only the closed claims from 2013 to 2015 . Using the empirical probabilities based on all closed claims or this adjusted sample led to almost no difference in the posterior distribution of the parameters.

A similar analysis is performed for the presence of expense for open claims. The estimated probabilities that a claimant file with $w_{i, j, \mathrm{op}}^{\star}=0$ and which is open since DP development periods at the evaluation date settles with $w_{i, j, \mathrm{op}}=0$ are shown in Table 2 herein. They vary with the gravity of the claim. 

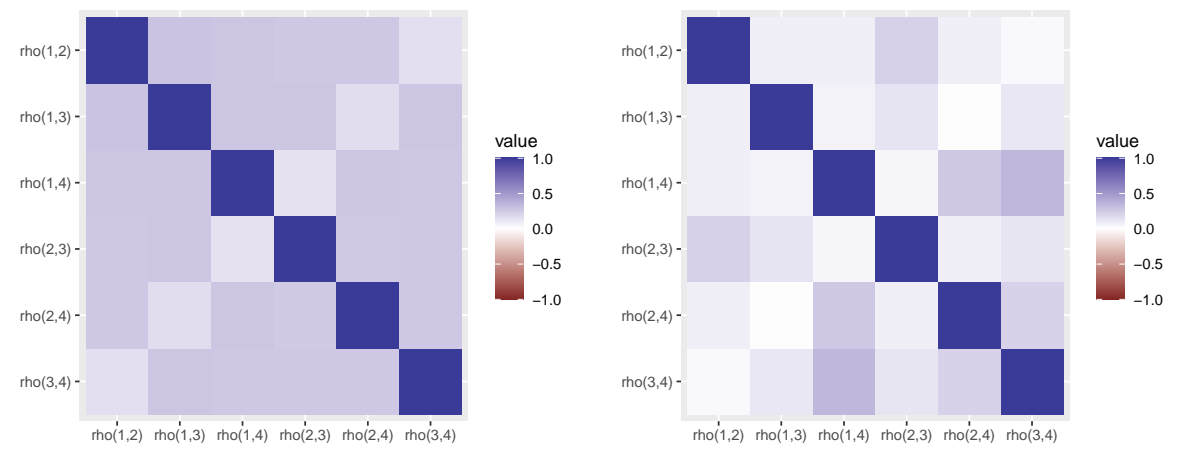

Figure 3: Heat maps of Kendall's tau matrix of the dependence parameters in $\Lambda$ for prior (left) and posterior (right) distributions.

Table 1: Probability (in \%) of staying in current category by gravity and development period, in the imputation models for type.

\begin{tabular}{rcccccccccc}
\hline & \multicolumn{2}{c}{$1-$ None } & \multicolumn{2}{c}{$2-$ M } & \multicolumn{2}{c}{$3-$ MI } & \multicolumn{2}{c}{$4-$ MC } & \multicolumn{2}{c}{$5-$ MIC } \\
DP & Minor & Major & Minor & Major & Minor & Major & Minor & Major & Minor & Major \\
\hline 1 & 49 & 32 & 77 & 42 & 83 & 55 & 85 & 78 & 93 & 96 \\
2 & 50 & 45 & 87 & 58 & 89 & 65 & 86 & 83 & 97 & 97 \\
3 & 58 & 58 & 90 & 67 & 92 & 73 & 89 & 86 & 98 & 97 \\
4 & 61 & 67 & 89 & 67 & 93 & 74 & 89 & 87 & 98 & 97 \\
5 & 69 & 74 & 88 & 67 & 92 & 74 & 90 & 88 & 96 & 97 \\
6 & 72 & 75 & 87 & 66 & 92 & 74 & 92 & 89 & 96 & 97 \\
7 & 69 & 76 & 85 & 65 & 91 & 76 & 91 & 89 & 98 & 97 \\
8 & 72 & 76 & 85 & 67 & 93 & 78 & 91 & 89 & 98 & 97 \\
9 & 72 & 78 & 85 & 70 & 93 & 79 & 91 & 89 & 98 & 97 \\
10 & 76 & 77 & 86 & 71 & 93 & 80 & 91 & 88 & 97 & 97 \\
11 & 74 & 82 & 87 & 71 & 94 & 78 & 91 & 87 & 97 & 97 \\
12 & 75 & 78 & 88 & 74 & 97 & 82 & 91 & 88 & 98 & 98 \\
13 & 100 & 100 & 89 & 75 & 95 & 82 & 93 & 90 & 100 & 98 \\
14 & 100 & 100 & 91 & 76 & 94 & 83 & 93 & 90 & 100 & 98 \\
15 & 100 & 100 & 93 & 78 & 92 & 81 & 93 & 90 & 100 & 98 \\
16 & 100 & 100 & 93 & 78 & 88 & 80 & 93 & 90 & 100 & 98 \\
17 & 100 & 100 & 92 & 78 & 93 & 76 & 93 & 89 & 100 & 98 \\
18 & 100 & 100 & 87 & 80 & 100 & 79 & 94 & 90 & 100 & 98 \\
19 & 100 & 100 & 88 & 82 & 100 & 77 & 97 & 89 & 100 & 97 \\
20 & 100 & 100 & 83 & 81 & 100 & 76 & 100 & 89 & 100 & 98 \\
\hline
\end{tabular}


Table 2: Probability (in \%) of staying in current category by gravity and development period, in the imputation models for the presence of external Expenses.

\begin{tabular}{rcc|ccc}
\hline DP & Minor & Major & DP & Minor & Major \\
\hline 1 & 88 & 70 & 11 & 98 & 98 \\
2 & 92 & 77 & 12 & 99 & 98 \\
3 & 95 & 83 & 13 & 99 & 99 \\
4 & 96 & 87 & 14 & 99 & 99 \\
5 & 97 & 91 & 15 & 100 & 99 \\
6 & 97 & 92 & 16 & 100 & 99 \\
7 & 97 & 94 & 17 & 100 & 99 \\
8 & 97 & 95 & 18 & 100 & 100 \\
9 & 98 & 97 & 19 & 100 & 100 \\
10 & 98 & 97 & 20 & 100 & 100 \\
\hline
\end{tabular}

\section{Models for missing covariates}

As it was chosen to perform the inference in two stages, i.e., (i) types and presence of expenses, and (ii) amounts given types and presence of expenses, the missing covariates models are also constructed separately for the two stages. This allows for a more precise modeling of the missing covariates for the amounts model, because the distribution of the covariates given that there was a strictly positive payment is different than over the entire dataset. In particular, the claim type is highly relevant to explain the pain level.

In the type model, only one covariate has missing values, namely the claimant's pain level on a scale of 1 to 10. In order to account properly for the relationship between this covariate and the others, a multinomial regression model is proposed as the prior for pain level. This model is consonant with the assumption that the pain level is missing at random (MAR).

The pain level is ordinal, so for simplicity a proportional odds regression model is assumed. If $Z$ denotes the pain level and $\mathbf{x}$ denotes the covariate vector, one has

$$
\operatorname{logit}\{\operatorname{Pr}(Z \geq j)\}=\alpha_{j}+\boldsymbol{\beta} \mathbf{x},
$$

where $\alpha_{j}$ is the intercept for category $j \in\{2, \ldots, 10\}$ and $\boldsymbol{\beta}$ is the vector of coefficients.

All the covariates that were used in the model for the type are also used in the model for the pain level, except the accident year which was unrelated to the pain level and added unnecessary noise. The parameters are given in Table 3.

For the amounts model, some covariates are missing for pain level, income level, and age. To simplify modeling, the pain level was assumed to be unrelated to income level and age. The data analysis supports this assumption, especially once the number of years that the policyholder has been insured with the company is in the model. As age and income level are related, however, income level was modeled based on other covariates, and then age was modeled conditional on income and the other covariates. 
Table 3: Parameters in the pain level proportional odds prior distribution for the types.

\begin{tabular}{lrlc}
\hline Covariate & Coefficient & Covariate & Coefficient \\
\hline (Intercept):2 & 2.3221 & After Reform & 0.3485 \\
(Intercept):3 & 1.5170 & Number of Injuries & 0.3957 \\
(Intercept):4 & 0.7281 & New Policy & 0.2588 \\
(Intercept):5 & 0.0873 & Policy age 1-2 & 0.1655 \\
(Intercept):6 & -0.7020 & Policy age 3-4 & 0.1248 \\
(Intercept):7 & -1.3141 & Married & 0.0073 \\
(Intercept):8 & -2.1705 & Divorced & 0.3037 \\
(Intercept):9 & -3.3772 & Other Civil Stat. & 0.1966 \\
(Intercept):10 & -4.4501 & Post-ref.: Major & 0.6953 \\
Major & -0.0842 & Post-ref.: Catastrophic & 2.1316 \\
Catastrophic & 0.7184 & Post-ref.: Number of Injuries & -0.2778 \\
\hline
\end{tabular}

The parameters for the pain level proportional odds model for the amounts are given in Table 4 below. All covariates that are relevant to pain level are considered, including claim type. The income indemnity level is ordinal with seven categories. Therefore, one can also call on a proportional odds regression model.

The number of years since the policyholder has been a client with the company was found to be a good proxy for age and was never missing, so although it was not used in the amounts model, it was included as a covariate. The parameters are reported in Table 5 below. For age, a Gaussian distribution was used; the parameters can be found in Table 6 herein.

\section{MCMC algorithm for type and presence of expenses}

The initial values for $s_{\mathrm{op}}$ and $w_{\mathrm{op}}$ are set at their censored values, and the missing covariates start at the mode in the sample of complete records. Based on these values, maximum likelihood estimation assuming no censoring is used to obtain initial values for $\beta^{S}, \beta^{W}$, and $\alpha^{W}$. The variance-covariance matrices $\Sigma_{m}$ and $\Sigma_{W}$ resulting from this procedure, multiplied by $(2.38)^{2} / d$, where $d$ is the dimension of the parameter being updated, are used as the proposal variance in the random walk Metropolis step. This factor has been shown to be optimal in some specific high-dimensional cases (Roberts and Rosenthal, 2001). The Metropolis-within-Gibbs algorithm goes as follows:

1. For $m \in\{2, \ldots, 5\}$, update the $d_{S}$-dimensional parameter $\beta_{m}^{S}$ corresponding to type $m$ :

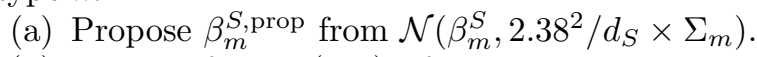

(b) Draw $U$ from $\mathcal{U}(0,1)$. If

$$
U \leq \pi_{0}\left(\beta_{m}^{S, \text { prop }}\right) / \pi_{0}\left(\beta_{m}^{S}\right) \times p\left(s \mid \beta_{m}^{S, \text { prop }}, \beta_{-m}^{S}, x\right) / p\left(s \mid \beta_{m}^{S}, \beta_{-m}^{S}, x\right),
$$

set $\beta_{m}^{S}=\beta_{m}^{T, \text { prop }}$; otherwise $\beta_{m}^{S}$ is unchanged. 
Table 4: Parameters in the pain level proportional odds prior distribution for the amounts.

\begin{tabular}{lrlr}
\hline Covariate & Coefficient & Covariate & Coefficient \\
\hline (Intercept):2 & 4.0379 & Newly Issued Policy & -0.0618 \\
(Intercept):3 & 2.9345 & Policy Aged 1-2 years & -0.1081 \\
(Intercept):4 & 1.9278 & Policy Aged 3-4 years & -0.0762 \\
(Intercept):5 & 1.1252 & Post-ref.: Major & 0.5133 \\
(Intercept):6 & 0.1922 & Post-ref.: Catastrophic & 1.5696 \\
(Intercept):7 & -0.4880 & Post-ref.: Number of Injuries & -0.0649 \\
(Intercept):8 & -1.4024 & Type M & -0.1928 \\
(Intercept):9 & -2.6606 & Type ME & 0.3188 \\
(Intercept):10 & -3.7338 & Type MI & 0.5397 \\
After Reform & 0.0572 & Type MIE & 0.5279 \\
Major & 0.0504 & Type MC & 0.7534 \\
Catastrophic & 0.3148 & Type MCE & 0.9680 \\
Number of Injuries & 0.1527 & Type MIC & 0.9923 \\
Legal Counsellor & 0.2751 & Type MICE & 1.3160 \\
Number of Years With Company & -0.0193 & & \\
\hline
\end{tabular}

Table 5: Parameters in the income indemnity level proportional odds prior distribution.

\begin{tabular}{lclr}
\hline Covariate & Coefficient & Covariate & Coefficient \\
\hline (Intercept):2 & 0.1643 & Number of Years With Company & 0.0133 \\
(Intercept):3 & -0.0804 & Newly issued policy & -0.0957 \\
(Intercept):4 & -0.4824 & Policy aged 1-2 years & 0.0806 \\
(Intercept):5 & -1.1376 & Policy aged 3-4 years & -0.0297 \\
(Intercept):6 & -1.9590 & Property Insurance & 0.0510 \\
(Intercept):7 & -3.4130 & Rating Score & -0.0153 \\
After Reform & -1.1468 & Type MIE & -0.1496 \\
Number of Injuries & 0.0072 & Type MIC & -0.2792 \\
Legal Counsellor & 0.4709 & Type MICE & -0.3351 \\
\hline
\end{tabular}


Table 6: Parameters in the age regression prior distribution.

\begin{tabular}{lrlr}
\hline Covariate & Coefficient & Covariate & Coefficient \\
\hline (Intercept) & 36.8701 & Rating score & -0.6039 \\
Indemnity [15,000-24,999] & -4.5280 & Major & 0.9448 \\
Indemnity [25,000-34,999] & -3.7669 & Catastrophic & -4.2750 \\
Indemnity [35,000-44,999] & -2.2049 & Optional Income & 2.6524 \\
Indemnity [45,000-54,999] & -0.2016 & Squared Rating Score & 0.0366 \\
Indemnity [55,000-74,999] & 2.1434 & Type M & 0.0849 \\
Indemnity Z 75000 & 3.6631 & Type ME & 1.4137 \\
Number of Injuries & 0.2339 & Type MI & -0.2718 \\
Legal Counsellor & -3.0878 & Type MIE & 2.1250 \\
Number of Years With Company & 0.4865 & Type MC & 3.1835 \\
Newly Issued Policy & -4.3840 & Type MCE & 3.3562 \\
Policy Aged 1-2 years & -2.0872 & Type MIC & 3.0282 \\
Policy Aged 3-4 years & -1.4046 & Type MICE & 3.6784 \\
Property Insurance & 1.4965 & & \\
\hline
\end{tabular}

2. Update the $d_{W}$-dimensional parameter $\left(\beta^{W}, \alpha^{W}\right)$ from the logistic model on the presence of Expenses with another Metropolis-Hastings step:

(a) Propose $\left(\beta^{W, \text { prop }}, \alpha^{W, \text { prop }}\right)$ from $\mathcal{N}\left[\left(\beta^{W}, \alpha^{W}\right), 2.38^{2} / d_{W} \times \Sigma_{W}\right]$.

(b) Draw $U$ from $\mathcal{U}(0,1)$. If

$$
U \leq \frac{p\left(w \mid \beta^{W, \text { prop }}, \alpha^{W, \text { prop }}, s, x\right) p i_{0}\left(\beta^{W, \text { prop }}, \alpha^{W, \text { prop }}\right)}{p\left(w \mid \beta^{W}, \alpha^{W}, s, x\right) \pi_{0}\left(\beta^{W}, \alpha^{W}\right)}
$$

set $\left(\beta^{W}, \alpha^{W}\right)=\left(\beta^{W \text {,prop }}, \alpha^{W \text {,prop }}\right)$; otherwise $\left(\beta^{W}, \alpha^{W}\right)$ is unchanged.

3. Update the censored values of the types and the presence of external expenses:

(a) Compute the probabilities (represented by dashed lines in Figure 5 of the main manuscript) as proportional to the probabilities from the model, given $x$.

(b) Draw $s_{\text {op }}$ and $w_{\text {op }}$ from the discrete distribution given by the transition probabilities.

4. Update the missing values of pain level. For each missing value, do the following:

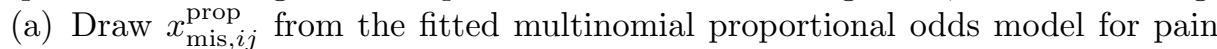
level given the other covariates (the prior and proposal distributions coincide).

(b) Draw $U$ from $\mathcal{U}(0,1)$. If

$$
U \leq p\left(s_{i j} \mid \beta^{S}, x_{\mathrm{obs}, i j}, x_{\mathrm{mis}, i j}^{\mathrm{prop}}\right) / p\left(s_{i j} \mid \beta^{S}, x_{\mathrm{obs}, i j}, x_{\mathrm{mis}, i j}\right),
$$

set $x_{\mathrm{mis}, i j}=x_{\mathrm{mis}, i j}^{\mathrm{prop}}$; otherwise, $x_{\mathrm{mis}, i j}$ is unchanged.

This algorithm can easily be coded in $\mathrm{R}$, and the proposals seem close to optimal as the acceptance rates were $18 \%$ for $\beta_{2}^{S}, \beta_{3}^{S}$, and $\beta_{4}^{S}, 17 \%$ for $\beta_{5}^{S}$, and $26 \%$ for the presence of Expenses parameters, which is reasonable in the present context. 

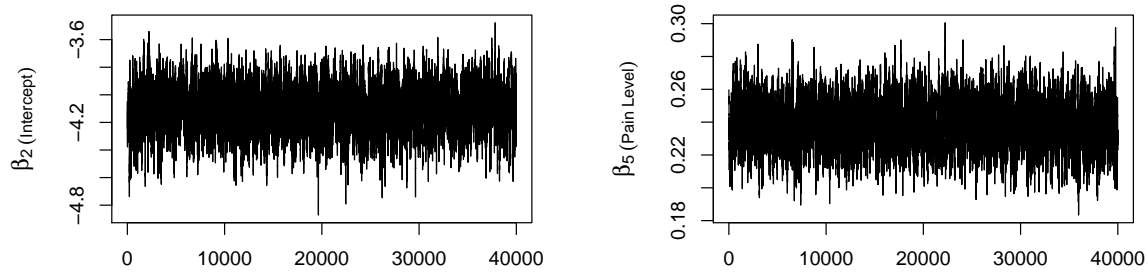

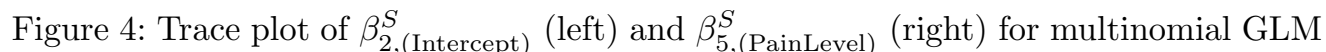
on type, with a burn-in period of 100,000, and 300,000 iterations thinning every fifth one.

Figure 4 above shows two typical trace plots: the intercept coefficient for Type 2 (left) and the pain level coefficient for Type 5 (right). Even though missing values for pain level were imputed, thereby increasing autocorrelation, the mixing is good on all parameters and the chain appeared to converge quickly. The posterior mean and standard deviations of the parameters are shown in Tables 7 and 8 below.

\section{MCMC algorithm for the amounts}

For the amounts model, a block Metropolis-within-Gibbs algorithm is used: the regression parameters are updated in blocks and the other shape, scale and dependence parameters are updated individually.

For the first 30,000 iterations, the proposal was based on the variance-covariance matrix obtained from the maximum likelihood estimation assuming no censoring. Mixing was very poor due to high autocorrelation for the regression coefficients, so for the subsequent 180,000 iterations, an adaptive proposal was used where the variance-covariance matrix of the proposals for the regression parameters was estimated from the run so far and updated every 50 iterations. As in the adaptive Metropolis-within-Gibbs algorithm of Roberts and Rosenthal (2009), the proposal variance for the individual parameter updates were tuned to lead to acceptance rates close to $44 \%$.

The adaptive algorithm improved greatly the behavior of the chain. The algorithm was then run with fixed proposal for 30,000 iterations. In this posterior sample, the autocorrelation was still large due to the random walk type of proposal, but it became clear that the posterior distributions of the parameters were close to a multivariate Normal. To improve mixing, the algorithm was finally run for another 30,000 iterations with a proposal in two cases: a random walk proposal with fixed covariance matrix for odd iterations, and an independent proposal with parameters estimated from the previous posterior sample for even iterations. The independent proposal clearly improved the chain's mixing. Typical trace plots are shown in Figure 5 herein. The final algorithm is as follows: 
Table 7: Posterior mean (standard deviation) of multinomial coefficients for type. Base category: minor 2004 claim, claimant is single, has no injury, and policy is in force since at least five years.

\begin{tabular}{lrrrr}
\hline Covariate & \multicolumn{1}{c}{ Type $2-\mathrm{M}$} & Type $3-\mathrm{MI}$ & Type $4-\mathrm{MC}$ & Type $5-\mathrm{MIC}$ \\
\hline Intercept & $-4.107(0.164)$ & $-6.510(0.405)$ & $-7.458(0.284)$ & $-7.663(0.448)$ \\
After Reform & $1.790(0.164)$ & $0.743(0.496)$ & $0.201(0.486)$ & $-1.518(1.221)$ \\
Major & $-0.517(0.052)$ & $-0.079(0.127)$ & $-0.417(0.065)$ & $-0.249(0.092)$ \\
Catastrophic & $-0.778(0.419)$ & $-0.576(0.617)$ & $0.913(0.361)$ & $2.411(0.332)$ \\
New Policy & $-0.017(0.043)$ & $0.260(0.067)$ & $0.429(0.059)$ & $0.424(0.076)$ \\
Pol. Age 1-2 yrs & $0.013(0.040)$ & $0.202(0.066)$ & $0.453(0.056)$ & $0.364(0.072)$ \\
Pol. Age 3-4 yrs & $0.047(0.047)$ & $0.234(0.079)$ & $0.307(0.068)$ & $0.240(0.090)$ \\
Married & $0.086(0.034)$ & $0.124(0.057)$ & $0.707(0.051)$ & $0.298(0.065)$ \\
Divorced & $0.097(0.078)$ & $0.074(0.121)$ & $0.742(0.101)$ & $0.285(0.134)$ \\
Other Civil Stat. & $0.322(0.057)$ & $0.362(0.095)$ & $0.797(0.087)$ & $0.518(0.108)$ \\
1 Injury & $3.236(0.148)$ & $2.791(0.399)$ & $3.772(0.258)$ & $3.694(0.431)$ \\
2 Injuries & $4.016(0.154)$ & $3.549(0.413)$ & $4.670(0.264)$ & $4.545(0.437)$ \\
3 Injuries & $4.199(0.161)$ & $3.952(0.417)$ & $5.179(0.268)$ & $5.200(0.439)$ \\
4 Injuries & $4.703(0.166)$ & $4.410(0.423)$ & $5.912(0.273)$ & $6.017(0.444)$ \\
Z 5 Injuries & $5.549(0.170)$ & $5.386(0.422)$ & $6.923(0.280)$ & $7.022(0.446)$ \\
Pain level (1-10) & $1.241(0.078)$ & $2.077(0.106)$ & $1.479(0.230)$ & $1.991(0.230)$ \\
Post-ref: Maj/Cat & $0.066(0.007)$ & $0.166(0.013)$ & $0.192(0.011)$ & $0.239(0.014)$ \\
Post-ref: 2-3 Inj. & $0.445(0.107)$ & $1.362(0.167)$ & $2.873(0.196)$ & $3.943(0.226)$ \\
Post-ref: 4 Inj. & $-3.508(0.169)$ & $-3.306(0.546)$ & $-7.689(0.999)$ & $-5.851(1.439)$ \\
Post-ref: $\geq 5$ Inj. & $-2.331(0.177)$ & $-1.779(0.521)$ & $-5.217(0.539)$ & $-4.053(1.256)$ \\
Year 2005 & $-0.422(0.079)$ & $-0.429(0.142)$ & $-0.155(0.113)$ & $-0.673(0.137)$ \\
Year 2006 & $-0.295(0.078)$ & $-0.570(0.153)$ & $0.078(0.112)$ & $-0.409(0.139)$ \\
Year 2007 & $0.087(0.082)$ & $-0.210(0.155)$ & $0.658(0.114)$ & $0.102(0.139)$ \\
Year 2008 & $0.339(0.089)$ & $-0.077(0.173)$ & $1.316(0.116)$ & $0.619(0.141)$ \\
Year 2009 & $0.607(0.087)$ & $-0.146(0.184)$ & $1.767(0.114)$ & $0.936(0.141)$ \\
Year 2010 & $0.509(0.073)$ & $-0.004(0.121)$ & $1.654(0.105)$ & $0.636(0.130)$ \\
\hline
\end{tabular}

1. For each $k \in\{1, \ldots, 4\}$, update the marginal parameters:

(a) For odd iterations, update $\beta_{k}^{Y}$ using a random walk proposal of the form $\mathcal{N}\left[\beta_{k}^{Y},(2.38)^{2} \Sigma_{k} / d_{k}\right]$, where $\Sigma_{k}$ is the covariance matrix from the adaptation run. The acceptance probability is

$$
\min \left\{1, \frac{p\left(y \mid \beta_{k}^{Y, \text { prop }}, \beta_{-k}^{Y}, \sigma, \xi, \gamma, \Lambda, x\right) \pi_{0}\left(\beta_{k}^{Y, \text { prop }}\right)}{p\left(y \mid \beta_{k}^{Y}, \beta_{-k}^{Y}, \sigma, \xi, \gamma, \Lambda, x\right) \pi_{0}\left(\beta_{k}^{Y}\right)}\right\} .
$$


Table 8: Posterior mean (standard deviation) of binomial coefficients for presence of Expenses. Base category: minor type 1 claims pre-reform for policy in force since at least one year.

\begin{tabular}{lc}
\hline Covariate & Expenses coefficient \\
\hline Intercept & $-2.999(0.052)$ \\
After Reform & $-0.373(0.059)$ \\
Major & $0.227(0.047)$ \\
Catastrophic & $0.895(0.230)$ \\
Newly Issued Policy & $0.435(0.033)$ \\
Post-ref: $\geq$ 5 Injuries & $1.074(0.049)$ \\
Post-ref: Major or Cat & $0.415(0.072)$ \\
Type 2 - M & $1.173(0.047)$ \\
Type 3 - MI & $2.265(0.059)$ \\
Type 4 - MC & $2.772(0.054)$ \\
Type 5 - MIC & $3.316(0.064)$ \\
\hline
\end{tabular}
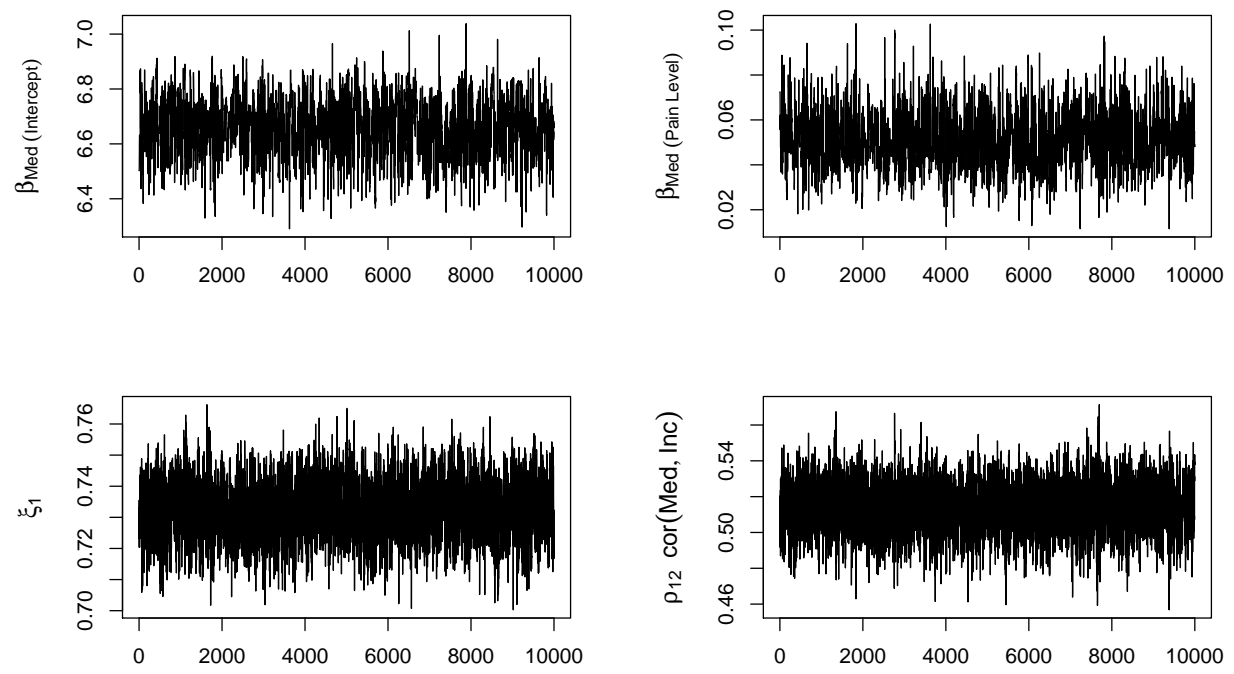

Figure 5: Trace plot of $\beta_{1, \text { (Intercept) }}^{Y}$ (top left), $\beta_{1,(\text { painlevel })}^{Y}$ (top right), $\xi_{1}$ (bottom left) and $\rho_{12}$ (bottom right), based on the final 30,000 iterations thinning every third one.

(b) For even iterations, update $\beta_{k}^{Y}$ using an independent Gaussian proposal $\mathcal{N}\left(\widehat{\mu}_{k}^{Y}, \widehat{\Sigma}_{k}^{Y}\right)$, where $\widehat{\mu}_{k}^{Y}$ and $\widehat{\Sigma}_{k}^{Y}$ are the mean and variance estimated from the sample generated in the fixed proposal run, respectively. The acceptance 
probability is

$$
\min \left\{1, \frac{p\left(y \mid \beta_{k}^{Y, \text { prop }}, \beta_{-k}^{Y}, \sigma, \xi, \gamma, \Lambda, x\right) \pi_{0}\left(\beta_{k}^{Y, \text { prop }}\right) \phi\left(\beta_{k}^{Y} ; \widehat{\mu}_{k}^{Y}, \widehat{\Sigma}_{k}^{Y}\right)}{p\left(y \mid \beta_{k}^{Y}, \beta_{-k}^{Y}, \sigma, \xi, \gamma, \Lambda, x\right) \pi_{0}\left(\beta_{k}^{Y}\right) \phi\left(\beta_{k}^{Y, \text { prop }} ; \widehat{\mu}_{k}^{Y}, \widehat{\Sigma}_{k}^{Y}\right)}\right\} .
$$

(c) Update $\lambda_{k}$ in a Metropolis-Hastings step with reflected Normal proposal $\mathcal{N}\left(\lambda_{k}, \sigma_{\lambda}\right)$.

(d) Update $\xi_{k}$ in a Metropolis-Hastings step with reflected Normal proposal $\mathcal{N}\left(\xi_{k}, \sigma_{\xi}\right)$.

2. Update the seven dependence parameters in a Gibbs sampler:

(a) Update $\rho_{i, \ell}$ using a random walk proposal $\mathcal{N}\left(\rho_{i, \ell}, \sigma_{\rho}\right)$ restricted to the domain where the covariance matrix stays positive definite. If $f(x)$ is the determinant of the correlation matrix when the elements $i, j$ and $j, i$ (to be updated) are replaced by $x$, the interval is obtained by solving the quadratic $a r^{2}+b r+c$, where $a=\{f(1)+f(-1)-2 f(0)\} / 2, b=\{f(1)-f(-1)\} / 2$ and $c=f(0)$.

(b) Update the between-claimant correlation $\gamma$ with a random walk MetropolisHastings step.

(c) Update the latent vector $a$ in one block, viz.

i. Draw $a^{\text {prop }} \sim \mathcal{N}\left(a, \sigma_{a}^{2} \mathbf{I}_{4 \times 4}\right)$. (When $\sigma_{a}^{2}=0.0039$, acceptance rates are around $22 \%$ ).

ii. Draw $U \sim \mathcal{U}(0,1)$. If $U \leq \pi_{0}\left(\Lambda, a^{\text {prop }}\right) / \pi_{0}(\Lambda, a)$ set $a=a^{\text {prop}}$; otherwise keep the previous value $a$.

3. Update the censored responses in a Gibbs sampler. For each open file:

(a) Draw $\mathbf{1}\left(T_{f} \leq t_{\text {now }}\right)$, i.e., whether it closes without further payment, using the Binomial model described in Section 4.2 of the main paper.

(b) If $\mathbf{1}\left(T_{f} \leq t_{\text {now }}\right)=1$, set $y_{\mathrm{op}, i j}=y_{\mathrm{op}, i j}^{\star}$.

(c) If $\mathbf{1}\left(T_{f} \leq t_{\text {now }}\right)=0$, for each $k$ such that $M_{i j k}=1$, draw $y_{\mathrm{op}, i j k}$ from the skewed normal model truncated at $y_{\mathrm{op}, i j k}^{\star}$ conditional on the other values of $y_{\mathrm{op}, i j,-k}$. This is done using a Metropolis-Hastings step with reflected Normal proposal.

4. Update sequentially the missing values of pain level, income indemnity level, and age. For each missing value, do the following:

(a) Draw $x_{\mathrm{mis}, i j}^{\mathrm{prop}}$ from the corresponding regression model for this covariate given the other covariates.

(b) Draw $U$ from $\mathcal{U}(0,1)$. If

$$
U \leq \frac{p\left(y_{i} \mid \beta^{Y}, \sigma, \xi, \gamma, \Lambda, x_{\mathrm{obs}}, x_{\mathrm{mis}, i j}^{\mathrm{prop}}\right)}{p\left(y_{i} \mid \beta^{Y}, \sigma, \xi, \gamma, \Lambda, x_{\mathrm{obs}}, x_{\mathrm{mis}, i j}\right)},
$$

set $x_{\mathrm{mis}, i j}=x_{\mathrm{mis}, i j}^{\mathrm{prop}}$; otherwise $x_{\mathrm{mis}, i j}$ is unchanged.

The evaluation of the amount likelihood is computationally challenging due to the different possible combinations of types for all claimants involved in a claim, $v_{i}$, leading to different copulas.

The posterior mean and standard deviation of the marginal parameters are given in Tables 9-10. The copula parameter estimation results are shown in Table 11. 


\section{References}

Barnard, J., McCulloch, R., and Meng, X.-L. (2000). "Modeling covariance matrices in terms of standard deviations and correlations, with application to shrinkage." Statistica Sinica, 10: 1281-1311. 3, 4

Roberts, G. O. and Rosenthal, J. S. (2001). "Optimal scaling for various MetropolisHastings algorithms." Statistical Science, 16: 351-367. 8

- (2009). "Examples of adaptive MCMC." Journal of Computational and Graphical Statistics, 18: 349-367. 11 
Table 9: Posterior mean (standard deviation) of parameters in skewed normal regressions for log paid amounts of Medical benefits and Income replacement benefits.

\begin{tabular}{|c|c|c|c|c|}
\hline \multirow[b]{2}{*}{ Intercept } & \multicolumn{2}{|c|}{ Medical } & \multicolumn{2}{|c|}{ Income } \\
\hline & 6.6698 & $(0.0975)$ & 6.6933 & $(0.1704)$ \\
\hline After reform & -0.3729 & $(0.0716)$ & 0.0128 & $(0.1579)$ \\
\hline Major & -0.0608 & $(0.0416)$ & -0.0004 & $(0.0806)$ \\
\hline Catastrophic & 2.2463 & $(0.2154)$ & 1.8186 & $(0.2860)$ \\
\hline reform:Major & 1.0490 & $(0.0624)$ & 0.9578 & $(0.1142)$ \\
\hline reform:Catastrophic & 0.8591 & $(0.3145)$ & 0.1095 & $(0.3872)$ \\
\hline Motorcycle/scooter & -0.3958 & $(0.1186)$ & & \\
\hline Rating score & -0.0056 & $(0.0022)$ & & \\
\hline Property insurance & -0.0782 & $(0.0259)$ & & \\
\hline Newly issued policy & 0.0195 & $(0.0397)$ & & \\
\hline Policy aged 1-2 years & 0.0452 & $(0.0346)$ & & \\
\hline Policy aged $3-4$ years & 0.0738 & $(0.0392)$ & & \\
\hline Legal Counsellor & 0.1740 & $(0.0403)$ & & \\
\hline Claimant age & 0.0030 & $(0.0011)$ & 0.0042 & $(0.0024)$ \\
\hline Initial \# of injuries & 0.1089 & $(0.0059)$ & 0.0700 & $(0.0134)$ \\
\hline Pain level & 0.8417 & $(0.0433)$ & 0.0591 & $(0.0167)$ \\
\hline Type ME & 0.5807 & $(0.0558)$ & & \\
\hline Type MI & 1.3297 & $(0.0565)$ & & \\
\hline Type MIE & 1.4079 & $(0.0498)$ & 0.6735 & $(0.0745)$ \\
\hline Type MC & 1.8520 & $(0.0519)$ & & \\
\hline Type MCE & 1.6956 & $(0.0733)$ & & \\
\hline Type MIC & 2.2962 & $(0.0574)$ & 0.6102 & $(0.0983)$ \\
\hline Type MICE & 0.0495 & $(0.0114)$ & 1.3959 & $(0.0832)$ \\
\hline Initial reserve & -0.0002 & $(0.0002)$ & 0.0007 & $(0.0010)$ \\
\hline Year before reform & 0.1211 & $(0.0105)$ & & \\
\hline Optional Income & & & 0.4687 & $(0.2296)$ \\
\hline Indemnity [15000 - 24999] & & & -0.0574 & $(0.1040)$ \\
\hline Indemnity [25000 - 34999] & & & 0.1276 & $(0.0868)$ \\
\hline Indemnity [35000 - 44999] & & & 0.2273 & $(0.0740)$ \\
\hline Indemnity [45000 - 54999] & & & 0.1750 & $(0.0841)$ \\
\hline Indemnity [55000 - 74999] & & & 0.0482 & $(0.0945)$ \\
\hline Indemnity $\geq 75000$ & & & 0.0112 & $(0.1680)$ \\
\hline$\lambda$ & 0.9260 & $(0.0141)$ & 0.5207 & $(0.0132)$ \\
\hline$\xi$ & 0.7323 & $(0.0089)$ & 0.7925 & $(0.0172)$ \\
\hline
\end{tabular}


Table 10: Posterior mean (standard deviation) of parameters in skewed normal regressions for log paid amounts for Caregiver benefits and the external Expenses.

\begin{tabular}{lrrrr}
\hline & \multicolumn{2}{c}{ Caregiver } & \multicolumn{2}{c}{ Expenses } \\
\hline Intercept & 6.4923 & $(0.2035)$ & 6.1820 & $(0.4973)$ \\
After reform & 0.0178 & $(0.2298)$ & -0.0260 & $(0.2124)$ \\
Major & 0.0733 & $(0.0673)$ & -0.0757 & $(0.1333)$ \\
Catastrophic & 3.4049 & $(0.2625)$ & 1.0973 & $(0.5702)$ \\
Reform:Major & 1.3058 & $(0.2268)$ & 0.6873 & $(0.1866)$ \\
Reform:Catastrophic & 0.4338 & $(0.4111)$ & 0.1319 & $(0.8348)$ \\
Motorcycle/scooter & -0.3073 & $(0.2123)$ & -0.3958 & $(0.4500)$ \\
Rating score & -0.0065 & $(0.0039)$ & -0.0169 & $(0.0067)$ \\
Newly issued policy & 0.0679 & $(0.0672)$ & & \\
Policy aged 1-2 years & 0.1444 & $(0.0588)$ & & \\
Policy aged 3-4 years & 0.1338 & $(0.0683)$ & & \\
Claimant age & 0.0037 & $(0.0022)$ & 0.0006 & $(0.0116)$ \\
Initial \# of injuries & 0.0678 & $(0.0125)$ & 0.0670 & $(0.0214)$ \\
Pain level & 0.0704 & $(0.0215)$ & 0.0385 & $(0.0344)$ \\
Type ME & & & 0.7722 & $(0.1653)$ \\
Type MIE & & & 1.2713 & $(0.1914)$ \\
Type MCE & 0.6110 & $(0.0627)$ & 1.2242 & $(0.1919)$ \\
Type MIC & -0.1854 & $(0.0870)$ & & \\
Type MICE & 0.4586 & $(0.0706)$ & 1.7699 & $(0.2125)$ \\
Initial reserve & -0.0005 & $(0.0005)$ & & \\
Year before reform & 0.1612 & $(0.0151)$ & & \\
$\lambda$ & 0.6159 & $(0.0159)$ & 0.5444 & $(0.0440)$ \\
$\xi$ & 0.8074 & $(0.0159)$ & 0.8434 & $(0.0332)$ \\
\hline
\end{tabular}

Table 11: Summary of the posterior distributions of the parameters of the $t$ copula.

\begin{tabular}{cccc}
\hline & Mean & Standard Deviation & $95 \%$ Credible Interval \\
\hline$\rho_{1,2}$ & 0.514 & 0.013 & $(0.49,0.54)$ \\
$\rho_{1,3}$ & 0.602 & 0.011 & $(0.58,0.62)$ \\
$\rho_{1,4}$ & 0.427 & 0.028 & $(0.40,0.49)$ \\
$\rho_{2,3}$ & 0.441 & 0.020 & $(0.40,0.48)$ \\
$\rho_{2,4}$ & 0.455 & 0.035 & $(0.41,0.54)$ \\
$\rho_{3,4}$ & 0.384 & 0.038 & $(0.35,0.44)$ \\
$\gamma$ & 0.364 & 0.016 & $(0.33,0.40)$ \\
\hline
\end{tabular}

